\title{
A Comparative Study of First-Order Reliability Method-Based Steepest Descent Search Directions for Reliability Analysis of Steel Structures
}

\author{
Hamed Makhduomi, ${ }^{1}$ Behrooz Keshtegar, ${ }^{2}$ and Mehdi Shahraki ${ }^{3}$ \\ ${ }^{1}$ Department of Civil Engineering, Saravan Branch, Islamic Azad University, Saravan, Iran \\ ${ }^{2}$ Department of Civil Engineering, University of Zabol, Zabol, Iran \\ ${ }^{3}$ Department of Civil Engineering, Zahedan Branch, Islamic Azad University, Zahedan, Iran \\ Correspondence should be addressed to Hamed Makhduomi; hamy.mk@gmail.com
}

Received 28 January 2017; Accepted 11 May 2017; Published 7 September 2017

Academic Editor: Sertong Quek

Copyright (C) 2017 Hamed Makhduomi et al. This is an open access article distributed under the Creative Commons Attribution License, which permits unrestricted use, distribution, and reproduction in any medium, provided the original work is properly cited.

Three algorithms of first-order reliability method (FORM) using steepest descent search direction are compared to evaluate the reliability index of structural steel problems which are designed by the Iranian National Building code. The FORM formula is modified based on a dynamic step size which is computed based on the merit functions named modified Hasofer-Lind and Rackwitz-Fiessler (MHL-RF) method. The efficiency of the gradient, HL-RF, and MHL-RF method was compared for a bar structure under tensile capacity, a multispan beam under bending capacity, a connection under tension load, and a column under axial force. The results illustrated that the MHL-RF method is more efficient than the HL-RF and gradient method. The designed steel components by the Iranian National Building code showed good confidence levels with the reliability index in the range from 2.5 to 3.0 .

\section{Introduction}

In real engineering systems, various uncertainties are produced in designing, constructing, and servicing phases of structural systems. The analytical modeling, poor acknowledgement from the analytical modeling, human factors, and data experiments from the input variables such as mechanical properties, load, failure mode, and geometrical dimensions of engineering systems are some kinds of uncertainties. These uncertainties can be considered based on probabilistic models and the reliability analysis can evaluate safety levels for engineering problems by using the failure probability. The analytical methods including first-order reliability method $[1$, 2], second-order reliability method [3], and moment methods [4] or simulation methods such as Monte Carlo simulation (MCS) [2], subset simulation [5], and weighted simulation [6] are generally used for estimating failure probabilities based on probabilistic model. The simulation methods are time-consuming approach for complicated real engineering structures [5-7]. However, the analytical methods are more efficient with appropriate accuracy for real structural problems in reliability analysis. The first-order reliability method is widely used in robust design, code calibration, load combination, and evaluating the confidence level of engineering systems due to its simplicity and efficiency $[8,9]$.

Hasofer and Lind (1974) proposed an iterative formula to search the most probable failure point (MPP) which is a point on the failure surface with minimum distance from origin in the standard normal space. Thus, the main effort of FORM is to search the MPP based on the following optimization model [10]:

$$
\begin{aligned}
\text { Minimize } & \beta=\left(\mathbf{U}^{T} \mathbf{U}\right)^{1 / 2} \\
\text { subjected to } & g(\mathbf{U})=0,
\end{aligned}
$$

in which $\beta$ is the reliability index and $g(\mathbf{U})$ is the limit state function or the performance function in normal standard space. Hasofer and Lind utilized the above model for normal variables where later Rackwitz and Flessler (1978) extended 
the iterative FORM formula based on the distribution information of the basic random variables; that is, it is briefly called HL-RF. The HL-RF method was improved by Liu and der Kiureghian (1991) using a merit function to enhance the convergence properties. Santosh et al. (2006) improved the HL-RF method based on Armijo rule. The convergence properties of HL-RF are improved based on the stability transformation method (STM) [8], the relaxed approach $[10,11]$, conjugate search direction $[7,12-14]$, and sufficient descent condition [13]. The STM [8] using small step size and relaxed HL-RF [11] using a dynamic relaxed factor less than 1 can provide stable results for highly nonlinear performance function but are formulated with complicated formulations [9]. The drawback of the STM is enhanced by the conjugate search direction with chaotic step size using logistic map $[7,13]$ and the directional search direction [15]. The new modified FORM formulas have been developed based on a complicated formulation to search MPP. Thus, efficiency, simplicity, and robustness can be applied to select an iterative FORM formula for reliability analysis of real engineering problems. Therefore, the present study is organized based on two main aims as follows:

(1) Utilize the FORM formula for reliability analysis of steel structures designed by Iranian National Building code.

(2) Develop a simple FORM formula to compute the reliability index of steel structures.

This study is organized based on estimating the failure probabilities or reliability indexes of steel components using three FORM algorithms including the HL-RF, gradient method, and a modified HL-RF method. The modified HL-RF is developed based on a dynamic step size where the proposed step size is adjusted based on the merit function. The normal and nonnormal random variables with log-normal and Gumbel distributions are implemented to simulate the statistical properties of the basic random variables in reliability analysis of steel components. Thus, a MATLAB code is developed to consider the statistical characteristics of basic random variables in performance functions which are extracted from Iranian National Building code. The HL-RF, gradient, and modified HL-RF are compared using four steel examples as a bar, a multispan beam, a connection, and a column. The effects of the applied loads are evaluated to obtain a reliable level with reliability index of 3 . Results indicate that the proposed MHL-RF method is as robust as the HL-RF and gradient method but is more efficient. The reliability indexes under the service load of these four steel components are obtained in range from 2.5 to 3 .

\section{First-Order Reliability Method (FORM)}

Failure probability of steel components is computed through a probabilistic model $(g(\mathbf{X}))$ that includes strength unreliability (elastic modulus, dimensions, Poisson index, ultimate tension, and yielding tension) and load (live or dead loads, load capacity). The failure probability can be approximated based on the reliability index $(\beta)$ in FORM as follows [8]:

$$
P_{f}=\int_{g(\mathbf{X}) \leq 0} \cdots \int f_{\mathbf{X}}(x) d \mathbf{X} \approx \Phi(-\beta),
$$

in which $P_{f}$ is failure probability, $g(\mathbf{X})$ is the limit state function which separated design domain into safe and failure domains using the basic random variables $\mathbf{X}$ as $g(\mathbf{X})<0$ is failure regain and $g(\mathbf{X})>0$ is safe regain. The main effort in the FORM is to search the maximum probable point (MPP, i.e., $\mathrm{U}^{*}$ ) which is a point on the limit state surface with minimum distance to organ into normal standard space. This distance is defined as the reliability index. Consequently, $\beta=\left\|\mathbf{U}^{*}\right\|$ [14]. In FORM, the random variables from original space $(\mathbf{X})$ should be transferred into normal standard space $(\mathbf{U})$ where these variables are independent with means of zero and standard deviations of one using Rosenblatt's transformation; that is, $u=\Phi^{-1}\left\{F_{X}(x)\right\}$ as follows [11]:

$$
\mathbf{U}=\frac{\left(\mathbf{X}-\mu_{\mathbf{X}}^{e}\right)}{\sigma_{\mathbf{X}}^{e}}
$$

in which $\mu_{\mathrm{X}}^{e}$ and $\sigma_{\mathrm{X}}^{e}$ are, respectively, equivalent mean and standard deviation of random variable $\mathbf{X}$. According to Rosenblatt's transformation, equivalent mean and standard deviation for no-normal variables are calculable as follows [10]:

$$
\begin{aligned}
\sigma_{x}^{e} & =\frac{1}{f_{X}(x)} \phi\left[\Phi^{-1}\left\{F_{X}(x)\right\}\right], \\
\mu_{x}^{e} & =x-\sigma_{x}^{e} \Phi^{-1}\left[F_{X}(x)\right],
\end{aligned}
$$

where $\phi$ and $\Phi$ are the normal probability distribution function and cumulative distribution function, respectively. $f_{X}(x)$ and $F_{X}(x)$ are, respectively, probability distribution function and cumulative distribution function of random variable at point $x$. For searching the MPP, there are various FORM algorithms such as Hasofer-Lind method [16, 17], stability transformation method $[8,15]$, and conjugate gradient $[9,12]$, finite-step length [18], relaxed HL-RF method [10, 13], and chaotic conjugate search direction $[7,13]$. The main effort to develop the FORM formula is to improve the efficiency and robustness of FORM. The HL-RF, gradient, and modified HLRF methods which are formulated using the steepest descent search direction are applied to find MPP.

2.1. HL-RF Method. The iterative formula of FORM can be described by the following relation:

$$
\mathbf{U}_{k+1}=\mathbf{U}_{k}+\alpha_{k} \mathbf{d}_{k}
$$

where $\alpha_{k}$ is step size. In HL-RF method, the step size is considered as 1 . $\mathbf{d}_{k}$ is search direction vector, which is computed as follows [1]:

$$
\mathbf{d}_{k}=\frac{\nabla^{T} g\left(\mathbf{U}_{k}\right) \mathbf{U}_{k}-g\left(\mathbf{U}_{k}\right)}{\nabla^{T} g\left(\mathbf{U}_{k}\right) \nabla g\left(\mathbf{U}_{k}\right)} \nabla g\left(\mathbf{U}_{k}\right)-\mathbf{U}_{k},
$$


in which $\nabla g\left(\mathbf{U}_{k}\right)$ is gradient vector of the limit state function $g()$ at point $\mathbf{U}_{k}$. By replacing (6) with (5) and considering the step size equivalent to 1 , the FORM formula-based HL-RF method is rewritten as follows:

$$
\mathbf{U}_{k}^{\mathrm{HL}}=\frac{\nabla^{T} g\left(\mathbf{U}_{k}\right) \mathbf{U}_{k}-g\left(\mathbf{U}_{k}\right)}{\nabla^{T} g\left(\mathbf{U}_{k}\right) \nabla g\left(\mathbf{U}_{k}\right)} \nabla g\left(\mathbf{U}_{k}\right) .
$$

This approach can be moved from a point on the feasible design region to MPP on the limit state surface; thus the constraint of the probabilistic model in (1) is satisfied at the final iteration of the HL-RF method when stable results are obtained.

2.2. Gradient Method. In gradient method, the probabilistic constraint of the optimization model in (1) should be satisfied at each iteration. Therefore, the iterative formula of the HL$\mathrm{RF}$ is adjusted based on the following search direction in gradient method [1]:

$$
\mathbf{d}_{k}=\frac{\nabla^{T} g\left(\mathbf{U}_{k}\right) \mathbf{U}_{k}}{\nabla^{T} g\left(\mathbf{U}_{k}\right) \nabla g\left(\mathbf{U}_{k}\right)} \nabla g\left(\mathbf{U}_{k}\right)-\mathbf{U}_{k} .
$$

In this method, the limit state function should be considered equal to zero at each iteration as $\nabla g\left(\mathbf{U}_{k}\right)=0$. Therefore, the probabilistic constraint of (1) can be satisfied using Newton method at each iteration by the following iterative formula:

$$
\mathbf{U}_{k+1}^{i+1}=\mathbf{U}_{k+1}^{i}-\frac{g\left(\mathbf{U}_{k+1}^{i}\right)}{\left\|\nabla g\left(\mathbf{U}_{k+1}\right)\right\|^{2}} \nabla g\left(\mathbf{U}_{k+1}\right) \text {. }
$$

The gradient and HL-RF methods are formulated based on steepest descent search direction based on (6) and (8). However, it needs an inner loop in order to satisfy the limit state function equal to zero, that is, $\nabla g\left(\mathbf{U}_{k}\right)=0$, based on the gradient approach. As seen, the amount of evaluating the limit state function in the gradient method is slightly increased compared to the HL-RF method with same evaluating of the gradient vectors. In addition, the HL-RF method is similar to the gradient method with step size of 1 for reliability analysis. These methods may provide unstable results for highly nonlinear reliability problems $[7,8,15,18]$. However, the gradient and HL-RF method are FORM algorithms with a fast convergence rate because the step size in these approaches is selected equivalent to one, while the step size in the modified versions of FORMbased steepest descent search direction such as improved HLRF [1, 19], RHL-RF [11], and STM [8] is given less than 1 to achieve the stabilization. Hence, the improved versions of HL-RF may enhance the robustness of FORM formula compared to HL-RF for highly nonlinear problems but these approaches are computationally inefficient for moderately nonlinear or linear performance functions [7]. The conjugate search direction can improve the robustness and efficiency of FORM compared to the improved HL-RF methods, but the FORM-based conjugate search direction was formulated with complex relations. In this study, a modified HL-RF is developed to improve the capability of FORM formula.

\section{Modified HL-RF Method}

According to (5), the step size and search direction are two affective parameters in the iterative FORM formula. The iterative FORM formula can be controlled based on step size to search MPP. Therefore, the iterative formula of modified HL-RF (MHL-RF) can be obtained as follows:

$$
\begin{aligned}
\mathbf{U}_{k+1} & =\mathbf{U}_{k}+\alpha_{k} \mathbf{d}_{k}, \\
\mathbf{d}_{k} & =\frac{\nabla^{T} g\left(\mathbf{U}_{k}\right) \mathbf{U}_{k}-g\left(\mathbf{U}_{k}\right)}{\nabla^{T} g\left(\mathbf{U}_{k}\right) \nabla g\left(\mathbf{U}_{k}\right)} \nabla g\left(\mathbf{U}_{k}\right)-\mathbf{U}_{k},
\end{aligned}
$$

where $\alpha_{k}$ is the adjusted step size. In this study, the step size of MHL-RF method in (10) can be dynamically adjusted in range from 1.5 to 0 . It is supposed that the step size is adjusted by the following merit function:

$$
\begin{aligned}
m\left(\mathbf{U}_{k}\right)= & \left\|\mathbf{U}_{k}-\frac{\nabla^{T} g\left(\mathbf{U}_{k}\right) \mathbf{U}_{k}}{\nabla^{T} g\left(\mathbf{U}_{k}\right) \nabla g\left(\mathbf{U}_{k}\right)} \nabla g\left(\mathbf{U}_{k}\right)\right\|^{2} \\
& +\frac{g\left(\mathbf{U}_{k}\right)^{2}}{g\left(\mathbf{U}_{0}\right)^{2}} .
\end{aligned}
$$

It is clear that the merit function is a positive value $m\left(\mathbf{U}_{k}\right) \geq 0$ and it is computed based on the previous results as well as the HL-RF method. The second term of this merit function is a positive dimensionless value that should be decreased for sequence iterations of MHL-RF to satisfy the constraint of the probabilistic optimization model in (1) as $g\left(\mathbf{U}_{k}\right)^{2}<$ $g\left(\mathbf{U}_{k-1}\right)^{2}$. If $m\left(\mathbf{U}_{k}\right)$ is equivalent to zero, then a fixed point is obtained or the MHL-RF is converged. Thus $g\left(\mathbf{U}_{k}\right)=0$ and $\mathbf{U}_{k}-\left(\nabla^{T} g\left(\mathbf{U}_{k}\right) \mathbf{U}_{k} / \nabla^{T} g\left(\mathbf{U}_{k}\right) \nabla g\left(\mathbf{U}_{k}\right)\right) \nabla g\left(\mathbf{U}_{k}\right)=0$ when $m\left(\mathbf{U}_{k}\right)=0$ (8); it is supposed that for $k \rightarrow \infty$ then $m\left(\mathbf{U}_{k}\right)=0$; this means that $\alpha_{k} \mathbf{d}_{k} \approx 0$; consequently, $\mathbf{U}_{k+1} \approx \mathbf{U}_{k}$; therefore, $\mathbf{U}_{k+1}$ is a fixed point and the proposed method is converged. It is assumed that $m\left(\mathbf{U}_{k}\right)<m\left(\mathbf{U}_{k-1}\right)$ thus $m\left(\mathbf{U}_{k}\right)=0$ for $k \rightarrow \infty$. Therefore, the step size is computed as follows:

$$
\alpha_{k+1}= \begin{cases}\frac{m\left(\mathbf{U}_{k-1}\right)}{m\left(\mathbf{U}_{k}\right)} \alpha_{k} & m\left(\mathbf{U}_{k}\right) \geq m\left(\mathbf{U}_{k-1}\right) \\ \alpha_{k} & m\left(\mathbf{U}_{k}\right)<m\left(\mathbf{U}_{k-1}\right),\end{cases}
$$

in which the initial step size is considered as 1.5 (i.e., $\alpha_{0}=1.5$ ). According to the above adaptive step size in (12), it can be concluded that $\alpha_{k+1} \leq \alpha_{k}$ and also, as it is mentioned, if $m\left(\mathbf{U}_{k}\right)<m\left(\mathbf{U}_{k-1}\right)$, then $\alpha_{k} \mathbf{d}_{k}<\alpha_{k-1} \mathbf{d}_{k-1}$; thus $\alpha_{k} \mathbf{d}_{k} \approx 0$ when $k \rightarrow \infty$. If $m\left(\mathbf{U}_{k}\right) \geq m\left(\mathbf{U}_{k-1}\right)$, then according to (12) $\alpha_{k+1}<\alpha_{k}$; thus for $k \rightarrow \infty, \alpha_{k+1} \rightarrow 0$ and $\alpha_{k} \mathbf{d}_{k} \approx 0$ for highly nonlinear problems. If $\alpha_{k} \mathbf{d}_{k} \approx 0$, then the new points are located on the previous point based on the modified HL$\mathrm{RF}$ method; thus robust results for reliability analysis are obtained. The step size may be obtained more than 1 when a linear or moderately concave limit state function is selected. It is clear that this method is as simple as the HL-RF method but the MHL-RF method may provide a higher convergence rate than the HL-RF for moderate performance functions. The algorithm of the modified HL-RF method to code in computer software can be presented using the following steps: 


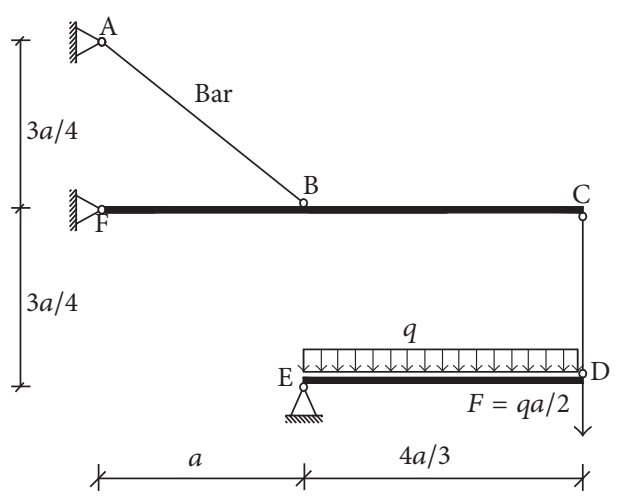

FIGURE 1: The steel bar structure with tensile performance function for Example 1.

(1) Define the limit state function $(g)$ and the convergence criterion $\left(\varepsilon=10^{-6}\right)$, statistical characteristics of basic random variables (mean, standard deviation, and distribution function for each random variable), and set $k=0, X_{0}=\mu$, and $\alpha_{0}=1.5$.

(2) Transfer the random variables from $X$-space to $U$ space according to (3) and (4).

(3) Compute the gradient vector and limit state function at point $\mathbf{U}_{k}$.

(4) Compute the merit function using (10) and the new adjusted step size based on (12), for $k \geq 2$.

(5) Compute the steepest descent search direction in terms of (6).

(6) Determine the new point according to (5).

(7) Transfer the basic random variables from $U$-space to $X$-space.

(8) Control the convergence criterion as $\mathbf{d}_{k}<\varepsilon$; if converged, stop and go to step (9); else, $k=k+1$ and go to step (2).

(9) Compute the reliability index and failure probability.

\section{Reliability Results and Comparative Examples}

According to three algorithms of HL-RF, gradient, and MHLRF, the reliability indexes of four moderately structural examples which were designed based on the Iranian National Building were evaluated to illustrate their performances in reliability analysis-based FORM. The numbers of gradient vector (iterations) for limit state functions which are computed using the central difference method as $\nabla g\left(\mathbf{U}_{k}\right)=$ $\left(g\left(\mathbf{U}_{k}+\Delta\right)-g\left(\mathbf{U}_{k}-\Delta\right)\right) / 2 \Delta$ and reliability indexes were considered as comparative criteria to compare the FORMbased steepest descent search directions which were coded in MATLAB using the stopping criterion as $\varepsilon=10^{-6}$.

4.1. A Bar under Axial Tensile Force. A structure under a uniform load on the beam ED which is shown in Figure 1 is supported using a bar FC and bar $\mathrm{AB}$ is connected at point $\mathrm{B}$ with bar $\mathrm{AB}$. The performance function for this structure is defined based on the tensile capacity of the bar $A B$ for this example.
TABLE 1: Random variables for the bar example under tensile load.

\begin{tabular}{lccc}
\hline Variable & Distribution & Mean & Standard deviation \\
\hline$q(\mathrm{t} / \mathrm{m})$ & Log-normal & 2 & 0.2 \\
$a(\mathrm{~m})$ & Log-normal & 3 & 0.2 \\
$A\left(\mathrm{~cm}^{2}\right)$ & Normal & 10 & 1 \\
$F_{u}\left(\mathrm{~kg} / \mathrm{cm}^{2}\right)$ & Normal & 100 & 400 \\
\hline
\end{tabular}

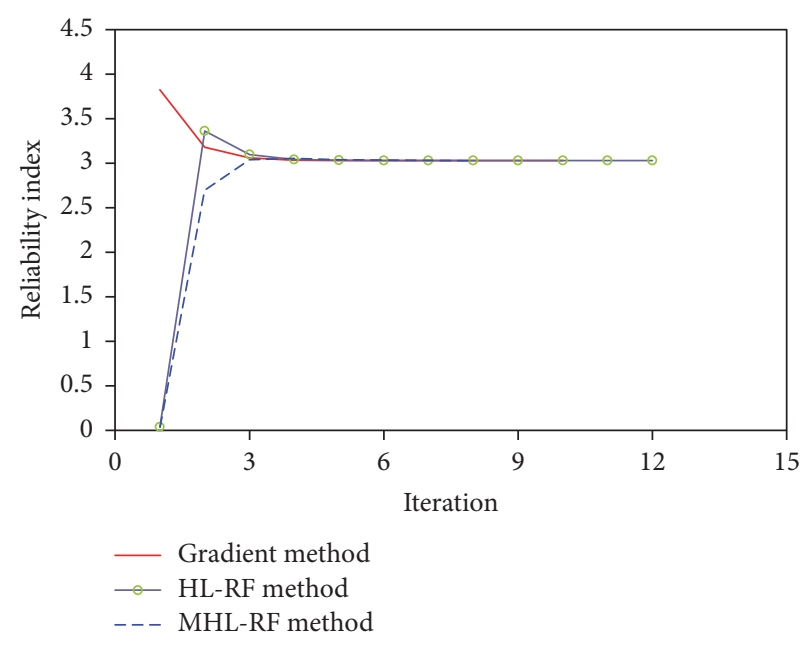

FIGURE 2: Comparison of reliability history for bar example.

According to the mechanical equations, the limit state function based on the capacity of bar $\mathrm{AB}$ under tensile force can be given as follows:

$$
g(\sigma)=\frac{245}{54} \frac{q a}{A}-F_{u},
$$

in which $a$ is a distance which is shown in Figure $1, A$ is the cross section of the bar AB, $q$ is the uniform applied load, and $F_{u}$ is ultimate strength of bar where the statistical characteristics of the random variables for this tensile component are given in Table 1.

The converged reliability index is $\beta=3.0277$ and the $\operatorname{MPP}$ is $\left[q, a, A, F_{u}\right]=[2.4714,3.2967,8.5955,3218.9558]$ based on the MHL-RF while the reliability index using Monte Carlo simulations (MCS) with $2 \times 10^{6}$ samples is 3.1124 . It can be concluded that the result of the proposed MHL$\mathrm{RF}$ for reliability index is a small difference with the MCS. Figure 2 illustrates the reliability index of different reliability methods using steepest descent search direction including gradient, HL-RF, and MHL-RF methods for bar example. It can be concluded that these three methods are robustly converted to same reliability index but the iterations to achieve stabilization are obtained differently. The HL-RF is more inefficient among other methods, that is, gradient and MHL-RF method, while MHL-RF is efficient method. The proposed MHL-RF method is converged using 8 iterations with a fast convergence rate compared to the other two methods.

The reliability indexes with respect to the ultimate strength and applied uniform load are shown in Figures 3 and 4 for bare example, respectively. It is obvious from 


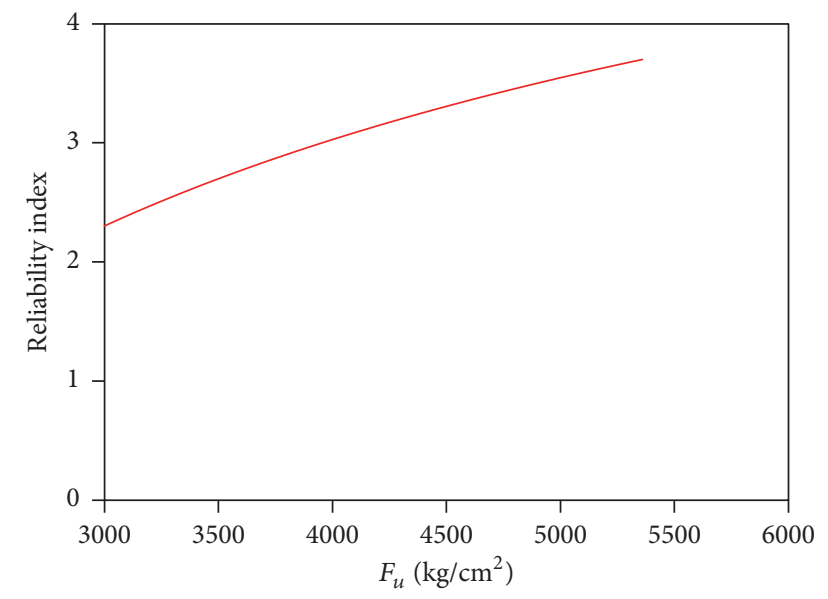

FIGURE 3: Reliability index with respect to ultimate strength $\left(F_{u}\right)$ for Example 1 .

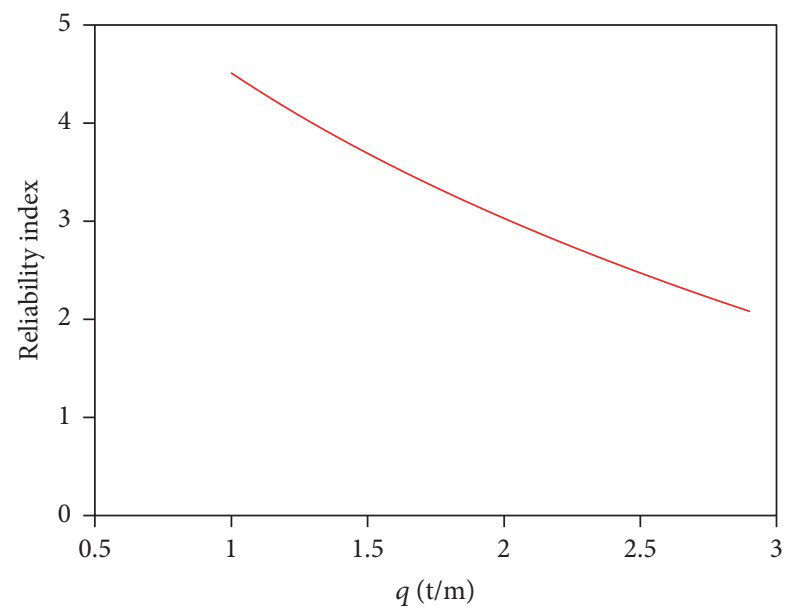

FIGURE 4: Reliability index with respect to load $(q)$ for Example 1.

Figures 3 and 4 that the reliability index is increased based on the increasing the ultimate strength while it is decreased with respect to increasing applied loads. The decreasing and increasing rate of reliability indexes are not obtained similarly for the same relative increment in the load and strength. It is clearly obvious from these figures that the reliability index of the steel bar is sensitive to the load compared to strength which is captured using the limit state function in (13).

4.2. Column under Buckling Force. A steel column under axial compressive load $P$ with length of $5 \mathrm{~m}$ is somatically plotted in Figure 5. The main failure mode of this column is the buckling capacity where the limit state function is given as follows:

$$
\begin{aligned}
& g=A F_{y}\left(1-\frac{\lambda^{2}}{2 C^{2}}\right)-P, \\
& C=\sqrt{\frac{2 \pi^{2} E}{F_{y}}}, \\
& \lambda=\frac{K L}{r}, \quad r=\sqrt{\frac{I}{A}} .
\end{aligned}
$$

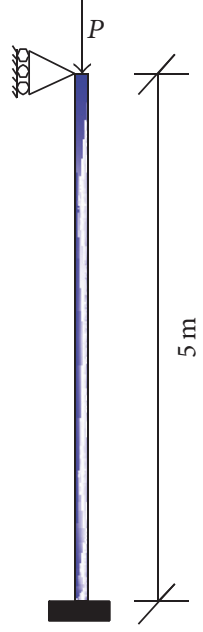

FIgURE 5: Schematic view of column.

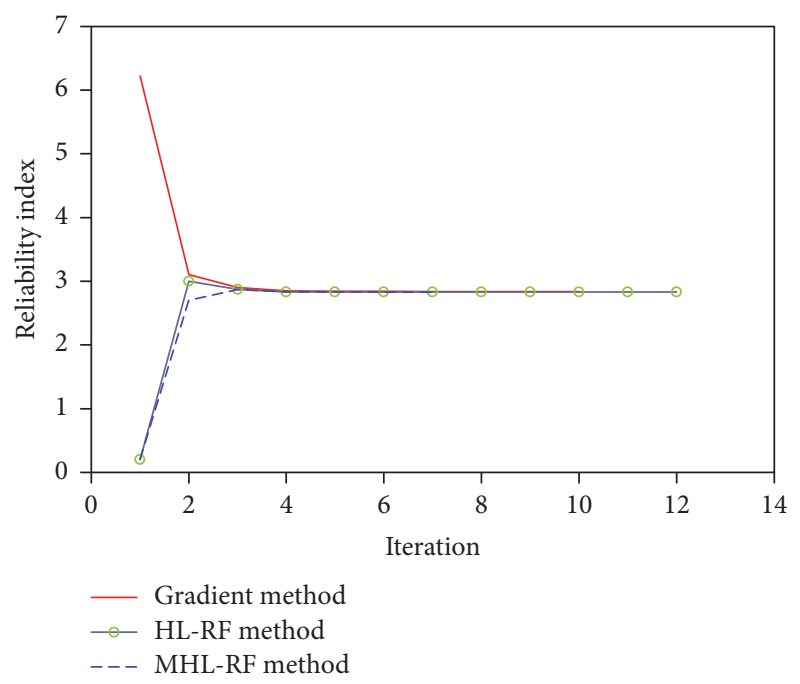

FIGURE 6: Reliability histories of different reliability method for column example.

Statistical characteristics of basic random variables for the limit state function in (14) are tabulated in Table 2.

The reliability index for this column is obtained as 2.8304 using HL-RF and MHL-RF method, $\beta=2.8403$ based on gradient method, and 2.7346 using MCS with $1.5 \times 10^{6}$ samples. The comparison of different reliability methods based on the iterative histories of reliability index is shown in Figure 6. It is obvious that the HL-RF is converged more inefficiently than the gradient and MHL-RF method, while the proposed MHL-RF method is converged, more efficiently. The total iterations for computing the gradient vectors of LSF using the MHL-RF method are about 3 and 6 iterations less than the gradient and HL-RF methods for this problem, respectively.

The reliability indexes are plotted with respect to the various applied compressive load $(P)$, yield strength $\left(F_{y}\right)$, and area of cross section $(A)$ based on the MHL-RF method in Figures 7-9, respectively. It is clear that the nonlinear 
TABLE 2: Statistical characteristics of random variables for the example of column.

\begin{tabular}{lcccc}
\hline Variable & Description & Mean & Standard deviation & Distribution \\
\hline$P(\mathrm{~kg})$ & Axial force & 70000 & 8000 & Gumbel \\
$A\left(\mathrm{~cm}^{2}\right)$ & Area of cross section & 65.3 & 5 & Normal \\
$F_{y}\left(\mathrm{~kg} / \mathrm{cm}^{2}\right)$ & Yield strength & 2400 & 200 & Log-normal \\
$E\left(\mathrm{~kg} / \mathrm{cm}^{2}\right)$ & Modulus of elasticity & $2 \times 10^{6}$ & $1 \times 10^{5}$ & Gumbel \\
$I\left(\mathrm{~cm}^{4}\right)$ & Second moment of inertia & 1360 & 136 & Log-normal \\
$K$ & Effective length factor & 0.7 & 0.12 & Log-normal \\
$L(\mathrm{~cm})$ & Length of column & 500 & 70 & Normal \\
\hline
\end{tabular}

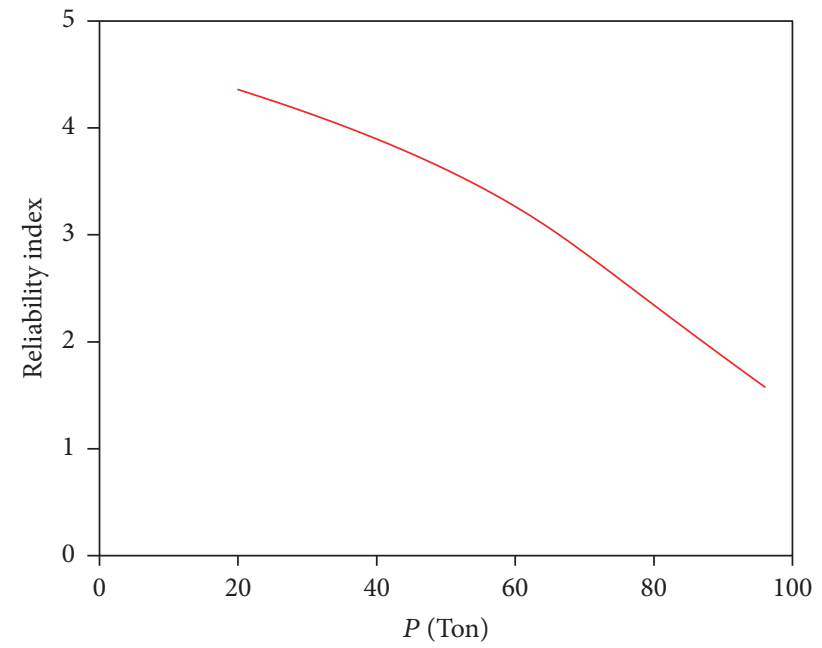

FIGURE 7: Reliability index with respect to the axial compressive load of column example.

relations are obtained between the basic random variables (i.e., $P, F_{y}$, and $A$ ) and the failure probabilities of column. The reliability index is increased by decreasing the load and increasing the $F_{y}$ and $A$. The applied compressive load is sensitive variable in compression with $F_{y}$ and $A$. The reliability index is more sensitive to the yield strength in the range from 1500 to $3000 \mathrm{~kg} / \mathrm{cm}^{2}$ and the area of cross section in the range between 40 and $90 \mathrm{~cm}^{2}$. It can be concluded that the decreasing load from 70 to 65 tons, increasing the yield strength from 2400 to $2650 \mathrm{~kg} / \mathrm{cm}^{2}$, and increasing cross section from 65.3 to $70.1 \mathrm{~cm}^{2}$ can improve the reliability index form 2.8 to 3 for this column example.

4.3. Two-Span Beam. A two-span beam with distributed load is considered for this example where schematic view of beam is shown in Figure 10. The limit state function under bending capacity is defined as follows:

$$
g=\varphi \frac{w l^{2}}{8 S}-k F_{y}
$$

in which $k$ and $\varphi$ are factors which indicate the effects of the maximum flexural strength under the external and the capacity of beam. The basic random variables of this example are given in Table 3.

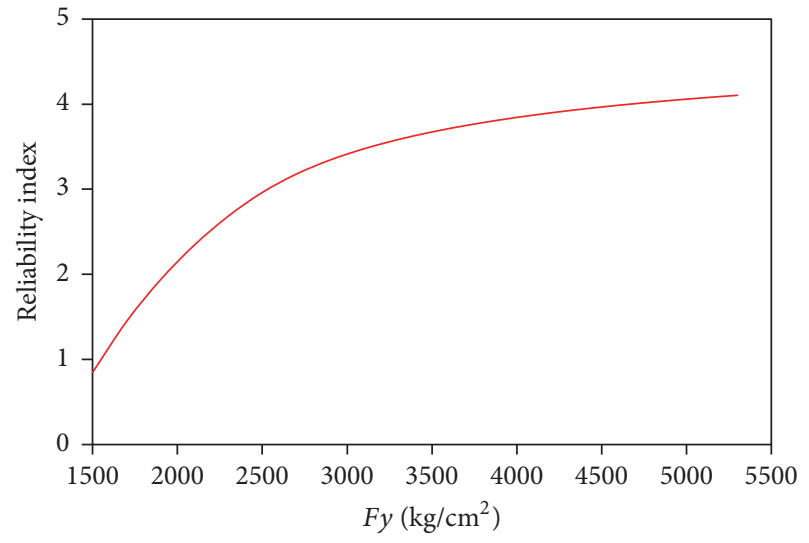

FIGURE 8: Reliability index with respect to the yield strength of column example.

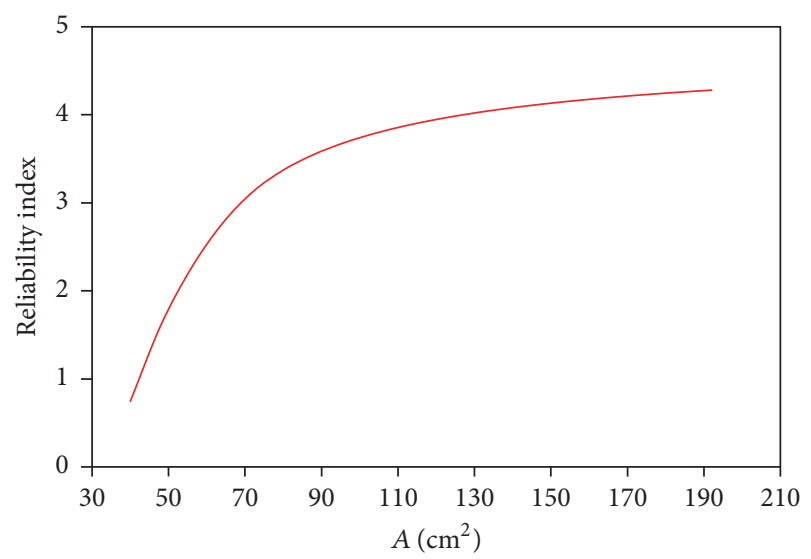

FIGURE 9: Reliability index with respect to the area of cross section of column example.

The reliability index and the MPP are, respectively, obtained as $\beta=2.4969$ and $\left[\varphi, W, L, S, K, F_{y}\right]=[0.9676$, $46.139,889.554,1984.295,0.97346,2225.507]$ and also the reliability index of 2.5132 is obtained using MCS with $2 \times$ $10^{6}$ samples. Figure 11 indicates the convergence histories of reliability index using HL-RF, gradient, and MHL-RF method for beam example. All reliability methods have been converged to stable reliability index but the proposed MHL$\mathrm{RF}$ method is more efficient. The proposed step size which is developed using the merit function can improve the efficiency 
TABLE 3: Random variables for two-span beam.

\begin{tabular}{lccc}
\hline Variables & Distribution function & Mean & Standard deviation \\
\hline$W(\mathrm{~kg} / \mathrm{cm})$ & Log-normal & 40 & 5 \\
$L(\mathrm{~cm})$ & Log-normal & 800 & 60 \\
$S\left(\mathrm{~cm}^{3}\right)$ & Normal & 2180 & 200 \\
$K$ & Normal & 1 & 0.1 \\
$F_{y}\left(\mathrm{~kg} / \mathrm{cm}^{2}\right)$ & Log-normal & 2400 & 200 \\
$\varphi$ & Normal & 0.9 & 0.08 \\
\hline
\end{tabular}

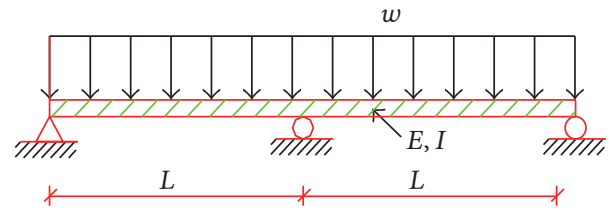

FIGURE 10: Schematic view of two-span beam.

of the FORM formula for moderately nonlinear performance functions. The proposed step size may be obtained as more than 1 for some of iterations in the MHL-RF method. Consequently, the convergence rate of the MHL-RF method is more than the HL-RF method, while its formulation is as simple as the HL-RF method and simpler than the gradient method.

Figures 12 and 13(a) illustrate the reliability indexes of two-span beam with respect to the various applied load $(W)$ and yield strength $\left(F_{y}\right)$, respectively. It is clear from results from Figures 12 and 13(a) that by increasing the load and decreasing the yield strength the reliability indexes are decreased. The load can be decreased by about $35 \mathrm{~kg} / \mathrm{cm}$ or the yield strength can be increased by about 2700 to improve the reliability index from 2.5 to 3 for this example.

4.4. Connection under Tensile Force. A connection according to Figure 13(b) under tensile force $T$ and bolts ultimate strength with type A325 and diameter $2 \mathrm{~cm}$ are considered for this example. This engineering system is utilized as a common connection in steel structures.

This structure includes various failure modes where they can be defined based on the failure of connected plates or bolts. Four limit state functions are defined for this example as follows:

(1) Tensile performance of thinner plates in the location of holes:

$$
g_{1}=2 A_{e} F_{u}-T
$$

(2) Tensile performance of thinner steel plates:

$$
g_{2}=2 A_{g} F_{y}-T \text {. }
$$

(3) Shear performance of bolts:

$$
g_{3}=\frac{32}{\sqrt{3}} A_{b} F_{u b}-T \text {. }
$$

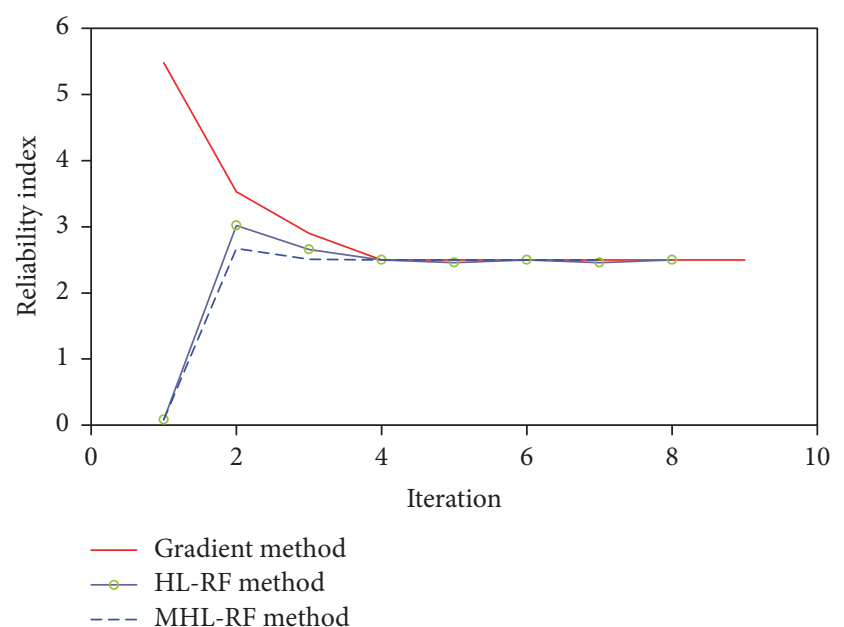

FIGURE 11: Convergence history of reliability method for two-span beam.

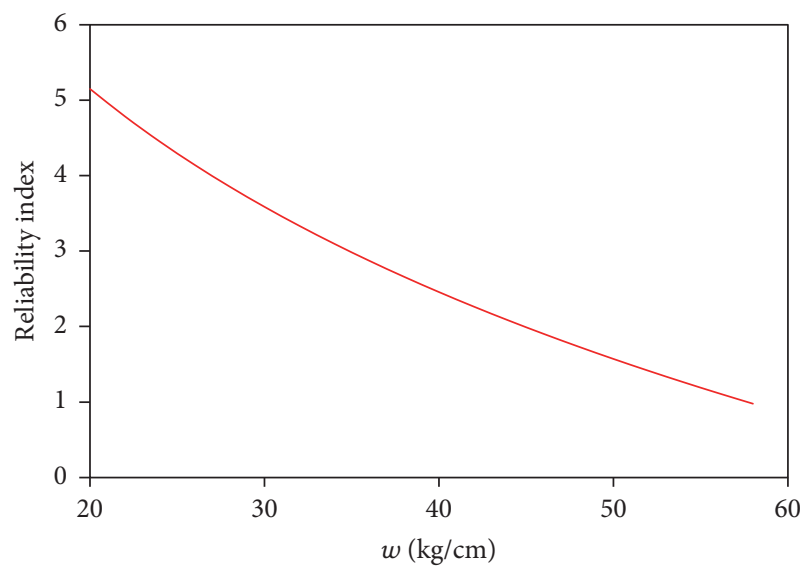

FIGURE 12: Reliability index with respect to the load for two-span beam example.

(4) Bearing performance of thinner plates:

$$
g_{4}=19.2 A_{k} F_{u}-T \text {. }
$$

The statistical characteristics of the basic random variables are tabulated in Table 4 for connection example.

According to the four presented limit state functions, the reliability indexes for this system connection are obtained as $\beta=\min \left(\beta_{1}, \beta_{2}, \beta_{3}, \beta_{4}\right)=\min (3.3442$, $3.1449,4.3374,4.3703)=3.1449$, while the reliability indexes of this example using MCS with $2.6 \times 10^{7}$ samples are $\beta=$ $\min (3.4642,3.1896,4.4219,4.3873)=3.1896$. It is clear that critical failure mode resulted based on the tensile failure mode of thinner steel plate, and the maximum reliability index is obtained based on the bearing the thinner steel plate. The reliability analysis is a suitable method to evaluate the safety levels or robust design of structures. This analysis method can show the effect of the important variables and the critical failure mode for design of structures. 


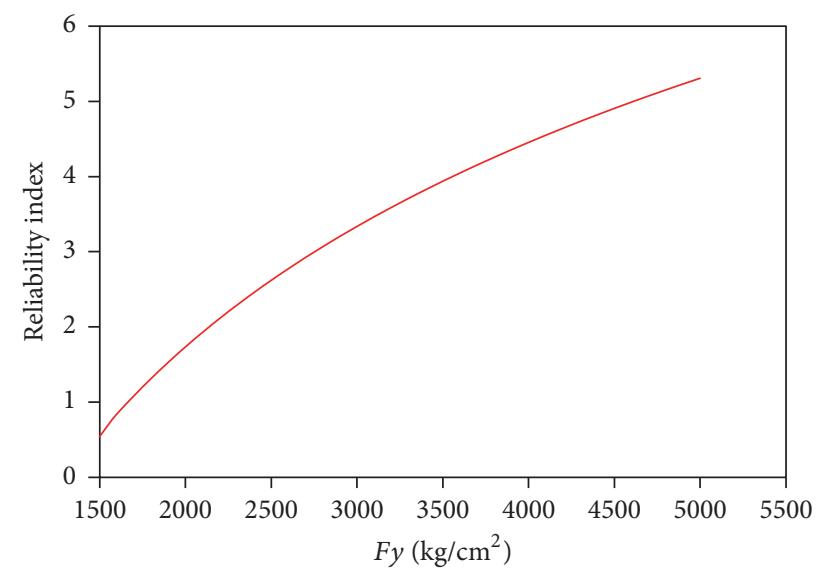

(a) Reliability index with respect to the yield strength for two-span beam example
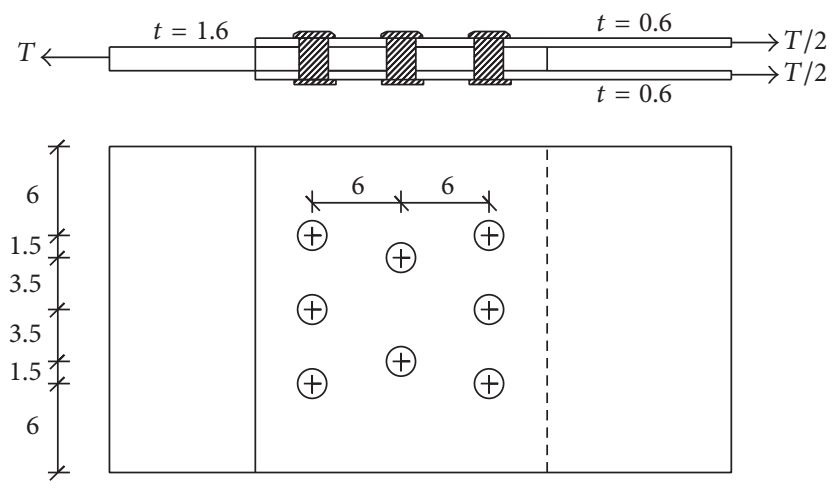

(b) Schematic view of the connection (sizes are in $\mathrm{cm}$ )

Figure 13

TABLE 4: Statistical characteristics of random variables for the connection example.

\begin{tabular}{lcccc}
\hline Variable & Description & Mean & Standard deviation & Distribution \\
\hline$T(\mathrm{~kg})$ & Applied tensile force & 37500 & 3750 & Gumbel \\
$F_{y}\left(\mathrm{~kg} / \mathrm{cm}^{2}\right)$ & Yield strength & 2400 & 200 & Log-normal \\
$F_{u}\left(\mathrm{~kg} / \mathrm{cm}^{2}\right)$ & Ultimate strength & 3700 & 350 & Log-normal \\
$F_{u b}\left(\mathrm{~kg} / \mathrm{cm}^{2}\right)$ & Ultimate strength of bolt & 8000 & 500 & Log-normal \\
$A_{e}\left(\mathrm{~cm}^{2}\right)$ & Effective cross section & 9.4 & 0.9 & Normal \\
$A_{g}\left(\mathrm{~cm}^{2}\right)$ & Total cross section & 13.2 & 1 & Normal \\
$A_{b}\left(\mathrm{~cm}^{2}\right)$ & Area of cross-sectional bolt & 3.14 & 0.3 & Normal \\
$A_{k}\left(\mathrm{~cm}^{2}\right)$ & Bearing cross section & 1.2 & 0.1 & Normal \\
\hline
\end{tabular}

The convergence histories of the minimum reliability index for four failure modes in (16)-(19) based on the HLRF, gradient, and MHL-RF methods are plotted in Figure 14 for Example 4. As seen, all reliability methods are converged to the same reliability index, but the iterations to achieve the stabilization are computed differently. The MHL-RF, HL$\mathrm{RF}$, and gradient methods produced the stable results after 7, 9, and 13 iterations. It is obvious that MHL-RF method is converged fast in all examples, and it is as simple as HL$\mathrm{RF}$ method. This method may provide stable results with a fast convergence rate for engineering problems where the convergence performances of FORM can be examined based on MHL-RF method for real complex problems in future. The proposed MHL-RF method can be implemented for steel code calibration-based Iranian National Building code of strength and load in future, successfully.

Figure 15 indicates the minimum reliability index based on the four failure modes in (16)-(19) of connection under various tensile forces. By increasing the load, the reliability index decreases. Also, the applied tensile load based on reliability index equal to 3 is about 40 tons. By comparing the load with reliability index 3 ( $T=40$ ton) and the applied load to design the connection ( $T=37.5$ ton), it is clear that the designed concoction-based Iranian code provided a suitable reliable level for this connection.

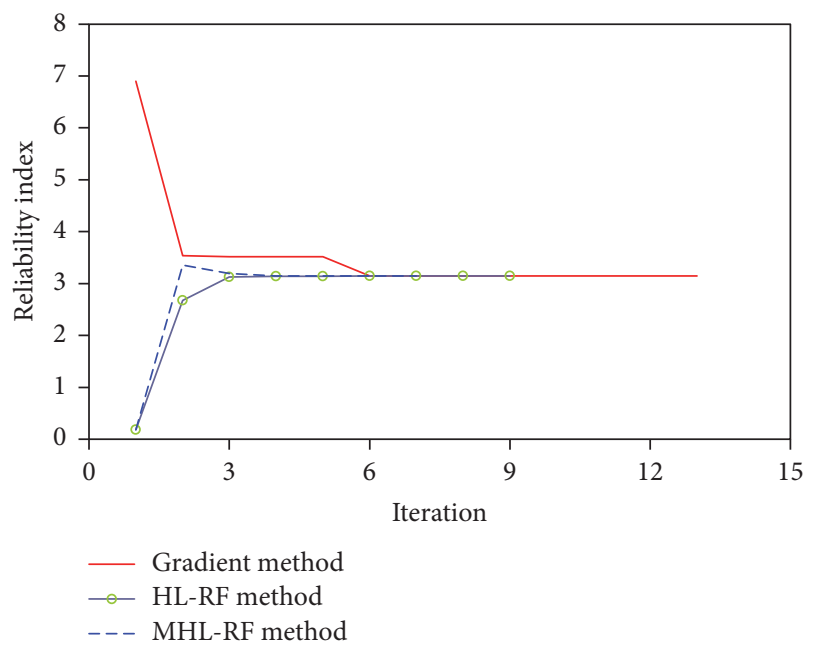

FIGURE 14: Convergence histories of reliability index for connection example.

\section{Conclusions}

In this study, first-order reliability algorithm-based HL-RF method is improved for reliability analysis of moderately 


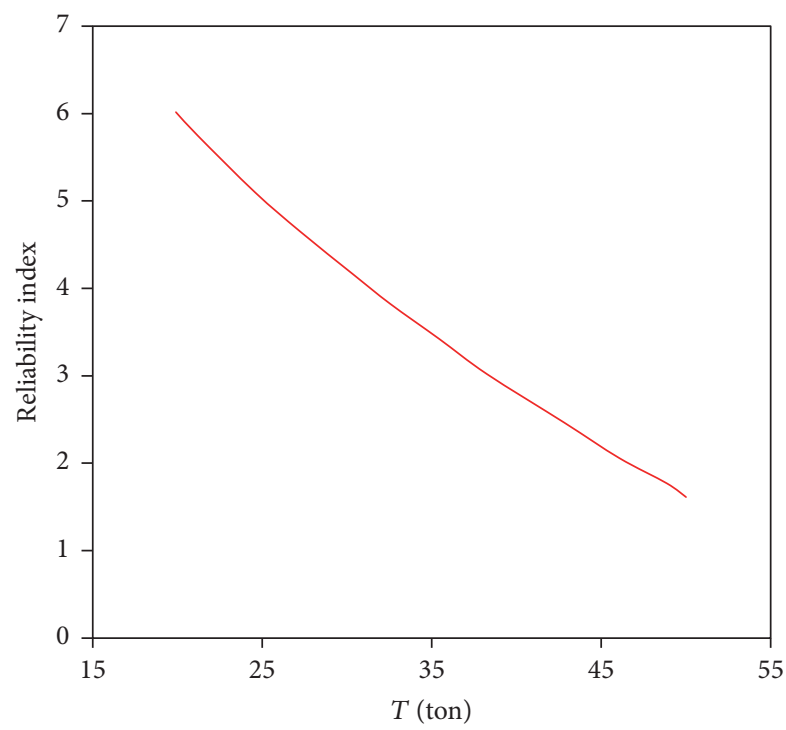

FIgURE 15: Minimum reliability index of the four failure modes of connection with respect to load.

nonlinear performance functions. A dynamic step size is proposed based on the merit function which is adjusted between 0 and 1.5 to improve the first-order reliability method (FORM) using steepest descent search direction. Three FORM algorithms including the HL-RF, gradient, and modified HL-RF (MHL-RF) method are compared with four moderate steel examples which are designed based on the Iranian National Building code including a bar under axial force, column under buckling forces, beam under bending capacity, and a connection under tensile load with multifailure modes. The results of the steel structures demonstrated that the MHL-RF method is as simple as the HL-RF method but is more efficient than the gradient and HL-RF methods for all examples. The proposed step size improved the efficiency of FORM formula; thus the MHL-RF can be used for reliabilitybased design optimization in future.

Results of reliability analysis for designed structures with Iranian National Building code can be summarized as follows:

(i) The reliability indexes of these components are obtained in the range from 2.5 to 3 .

(ii) By increasing load, the reliability index decreases, more affectively. However, the increasing strength capacity such as the yield strength can improve the reliability index.

(iii) Reliability index for beam (i.e., $\beta=2.5$ ) is obtained less than bar, column, and connection engineering reliability problems, while the connection example shows a maximum confidence level compared to other examples with reliability index about 3.15.

(iv) To determine the reliable levels, the reliability analysis-based MHL-RF method can be used to calibrate the code design in future.

\section{Conflicts of Interest}

The authors declare that they have no conflicts of interest.

\section{Acknowledgments}

The authors would like to acknowledge Islamic Azad University, Saravan, for funding the research scheme.

\section{References}

[1] P.-L. Liu and A. der Kiureghian, "Optimization algorithms for structural reliability," Structural Safety, vol. 9, no. 3, pp. 161-177, 1991.

[2] O. Ditlevsen and H. O. Madsen, Structural Reliability Methods, John Wiley and Sons, 1996.

[3] A. Der Kiureghian and M. de Stefano, "Efficient algorithm for second-order reliability analysis," Journal of Engineering Mechanics, vol. 117, no. 12, pp. 2904-2923, 1991.

[4] Y.-G. Zhao and Z.-H. Lu, "Fourth-moment standardization for structural reliability assessment," Journal of Structural Engineering, vol. 133, no. 7, pp. 916-924, 2007.

[5] S.-K. Au and J. L. Beck, "Estimation of small failure probabilities in high dimensions by subset simulation," Probabilistic Engineering Mechanics, vol. 16, no. 4, pp. 263-277, 2001.

[6] M. Rashki, M. Miri, and M. A. Moghaddam, "A new efficient simulation method to approximate the probability of failure and most probable point," Structural Safety, vol. 39, pp. 22-29, 2012.

[7] B. Keshtegar, "Stability iterative method for structural reliability analysis using a chaotic conjugate map," Nonlinear Dynamics, vol. 84, no. 4, pp. 2161-2174, 2016.

[8] D. Yang, "Chaos control for numerical instability of first order reliability method," Communications in Nonlinear Science and Numerical Simulation, vol. 15, no. 10, pp. 3131-3141, 2010.

[9] B. Keshtegar and M. Miri, "Introducing conjugate gradient optimization for modified HL-RF method," Engineering Computations, vol. 31, no. 4, pp. 775-790, 2014.

[10] A. Mohammadi Farsani and B. Keshtegar, "Reliability analysis of corroded reinforced concrete beams using enhanced HL-RF method," Civil Engineering Infrastructures Journal, vol. 48, no. 2, pp. 297-304, 2015.

[11] B. Keshtegar and M. Miri, "An enhanced HL-RF Method for the computation of structural failure probability based on relaxed approach," Civil Engineering Infrastructures Journal, vol. 1, no. 1, pp. 69-80, 2013.

[12] B. Keshtegar and M. Miri, "Reliability analysis of corroded pipes using conjugate HL-RF algorithm based on average shear stress yield criterion," Engineering Failure Analysis, vol. 46, pp. 104-117, 2014.

[13] B. Keshtegar, "Chaotic conjugate stability transformation method for structural reliability analysis," Computer Methods in Applied Mechanics and Engineering, vol. 310, pp. 866-885, 2016.

[14] B. Keshtegar, "Limited conjugate gradient method for structural reliability analysis," Engineering with Computers, vol. 33, no. 3, pp. 621-629, 2017.

[15] Z. Meng, G. Li, D. Yang, and L. Zhan, "A new directional stability transformation method of chaos control for first order reliability analysis," Structural and Multidisciplinary Optimization, vol. 55, no. 2, pp. 601-612, 2017.

[16] A. M. Hasofer and N. C. Lind, "Exact and invariant second moment code format," Journal of the Engineering Mechanics Division, vol. 111, no. 21, pp. 111-121, 1974. 
[17] R. Rackwitz and B. Flessler, "Structural reliability under combined random load sequences," Computers and Structures, vol. 9, no. 5, pp. 489-494, 1978.

[18] J.-X. Gong and P. Yi, "A robust iterative algorithm for structural reliability analysis," Structural and Multidisciplinary Optimization, vol. 43, no. 4, pp. 519-527, 2011.

[19] T. V. Santosh, R. K. Saraf, A. K. Ghosh, and H. S. Kushwaha, "Optimum step length selection rule in modified HLRF method for structural reliability," International Journal of Pressure Vessels and Piping, vol. 83, no. 10, pp. 742-748, 2006. 


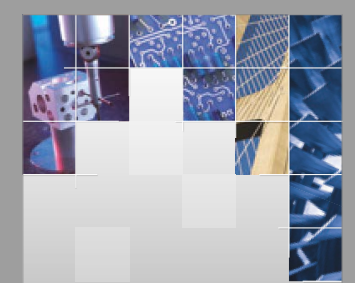

\section{Enfincering}
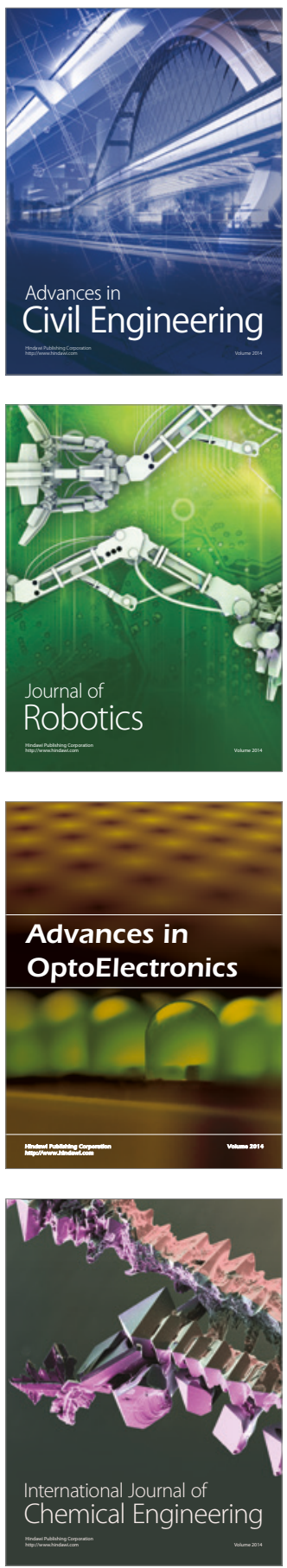

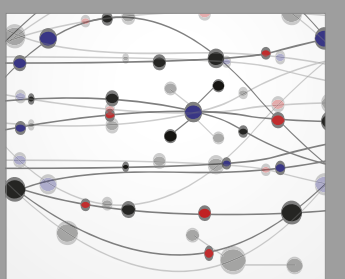

The Scientific World Journal

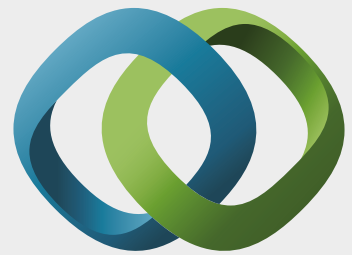

\section{Hindawi}

Submit your manuscripts at

https://www.hindawi.com
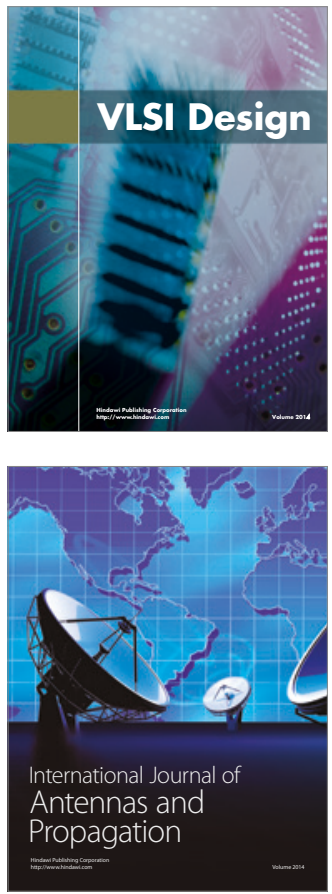

\section{Rotating}

Machinery
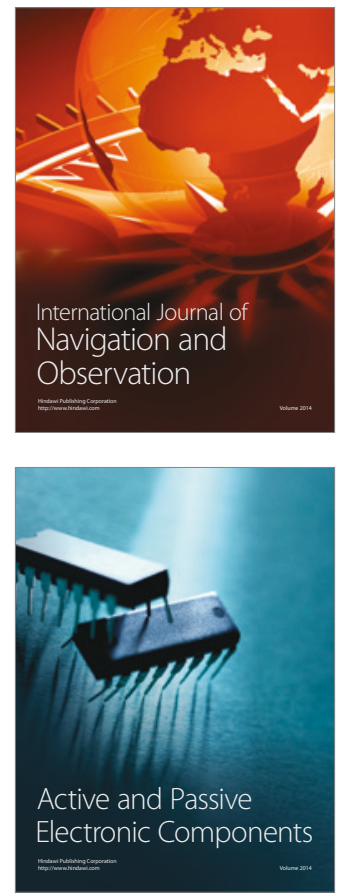
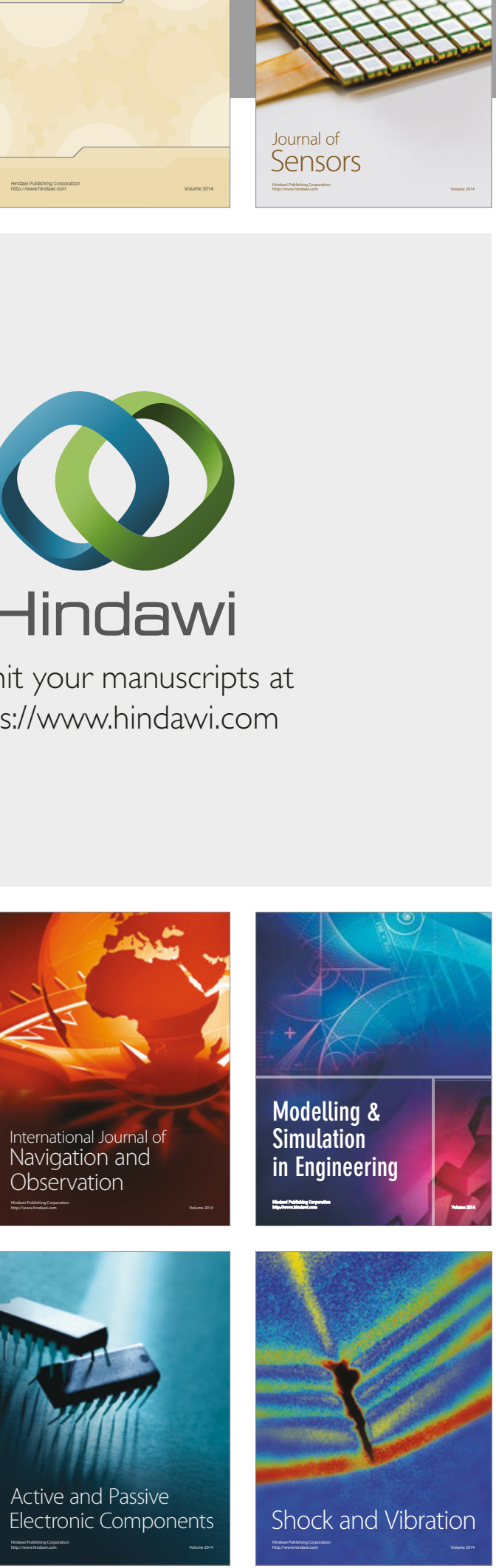
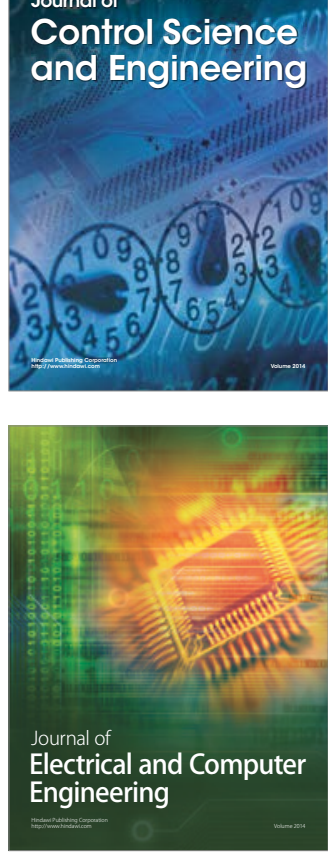

Distributed

Journal of

Control Science

and Engineering
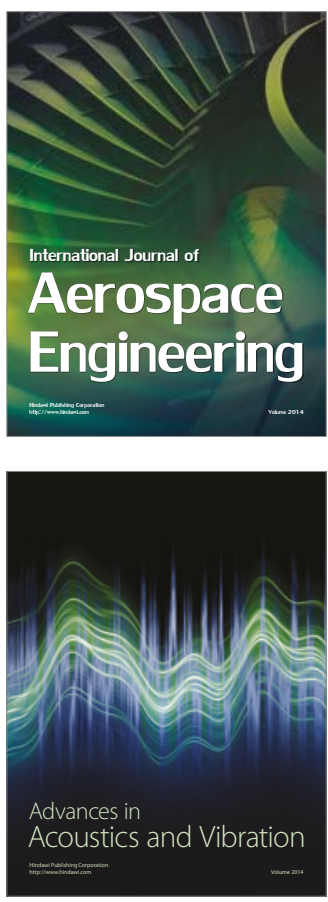

Sensor Networks 